\title{
Psoas Abscess after Radical Abdominal Hysterectomy: A Case Report and Review of the Literature
}

\author{
Dimitrios Spiliopoulos ${ }^{*}$, Khadra Galaal, Keith Godfrey and Raj Naik
}

Northern Gynaecological Oncology Centre, Queen Elizabeth Hospital, Gateshead, UK

\begin{abstract}
We report a case of a 38-year- old woman who presented with right lower quadrant abdominal pain and occasional fevers, five weeks after radical abdominal hysterectomy and pelvic node dissection for cervical carcinoma. After initial empirical antibiotic treatment failure, a decision was made to proceed with percutaneous drainage of a right psoas abscess. The antibiotic regimen was adjusted according to the culture results from the abscess. The patient's condition improved in the next 36 hours. The patient was discharged six days later on oral antibiotic treatment for four weeks.

Psoas abscess is a rare condition in general obstetrics and gynecology and its incidence in gynecological oncology is very low. This is a potentially fatal condition with difficult diagnosis and treatment. Appropriate management of these cases should include suspicion and confirmation of the diagnosis and aggressive medical and surgical treatment.
\end{abstract}

Keywords: Cervical carcinoma, psoas abscess, radical hysterectomy.

\section{INTRODUCTION}

First described by Minter in 1881, psoas abscesses can develop from hematogenous or lymphatic dissemination of an infection from an occult primary source (primary abscess) [1-3]. Direct spread from an adjacent infection of the bowel, the genital or urinary tract and the bone is another mechanism (secondary abscess). It may be uncertain whether involvement of an adjacent structure is a cause or a consequence of the abscess [4].

Primary abscesses are more common in tropical and developing countries. In Europe and North America 17 to 61 percent of the abscesses are primary, however, in Asia and Africa 99 percent are primary [3]. In some occasions it can be difficult to differentiate between primary and secondary cause [5].

Risk factors for the formation of primary abscesses are conditions associated with immunosuppression including cancer, diabetes, human immunodeficiency virus (HIV) infection, intravenous drug use, renal failure and chronic steroid use $[2,6]$. The case described was treated for cervical cancer, a condition that can potentially predispose to suppressed immune status and the development of sepsis in advanced stage.

Psoas abscesses are more common in males than females $[3,7]$ with a median age of 44 to 58 years in developed countries. They occur with the same frequency on the right and left side, bilateral abscesses being uncommon (one to five percent).

\footnotetext{
*Address correspondence to this author at the Gateshead Health NHS Foundation Trust, Northern Gynaecological Oncology Centre, Queen Elizabeth Hospital, Sheriff Hill, Gateshead, NE9 6SX, UK; Tel: (0044) 01914820000; Fax: 004401914456192; E-mail: dimspiliop@yahoo.com
}

Primary psoas abscesses are more frequently caused by a single organism [8]. Staphylococcus aureus has been the most commonly identified microbial organism associated with psoas abscess as it is present in $88 \%$ of the cases, together with Streptococcus (5\%) and E. coli (3\%). Bacteroides, Enteric pathogens, Streptococcus and Staphylococcus can also be present [3, 9-12]. Mycobacterium tuberculosis is still common in developing countries, but also frequently encountered in HIV infected patients. Secondary psoas abscesses can be monomicrobial or polymicrobial, including enteric organisms.

Psoas abscess is a condition rarely reported in the available obstetric and gynecologic literature. A few cases have been diagnosed after vaginal delivery [13-16], two more were reported during the course of a normal pregnancy [17, $18]$ and one case was reported after dilation and curretage for abortion with an intrauterine device in place [19].

In gynecological oncology it is very rarely encountered, despite the extensive tissue dissection and degree of radicality involved in many of these procedures. The clinical picture can be variable and nonspecific. It can present with the clinical triad of fever, back pain and groin or thigh pain, although the full triad is present in less than one third of the patients.

We present the first reported case of psoas abscess after radical hysterectomy and pelvic lymph node dissection for treatment of cervical cancer.

\section{CASE PRESENTATION}

A 38 year old G4P2 woman diagnosed with a stage $1 \mathrm{~b} 1$ poorly differentiated adenosquamous cervical carcinoma was treated in our institution with radical hysterectomy and bilateral systematic pelvic lymph node dissection. We performed the lymph node dissection after the uterus was 
removed. The extent of lymphadenectomy consisted of the removal of all visible pelvic lymph nodes from the bifurcation of the common iliac artery caudally. No enlarged common iliac or paraortic nodes were identified. The fascia of the iliopsoas muscle was incised along the line of the external iliac artery. The incision was carried up to the retractor, and the lateral external iliac nodes lying under the inguinal ligament were excised across the vessels. Small lymphatic channels and blood vessels were clipped with liga clips. The dissection continued along the external iliac artery and a sheet of fascia and nodal tissue was developed leaving the arteries clean from the bifurcation of the common iliac artery to the inguinal ligament. At the upper end of the dissection, the sheet of tissue was removed across the internal iliac artery. At the final stage, the dissection continued below the external iliac vein, emptying the obturator fossa of all nodes. The block of tissue containing lyphnodes was lifted out. Hemostasis at the end of the operation was meticulous using argon beam coagulation, no pelvic drains were used and the estimated blood loss was $600 \mathrm{ml}$. The vaginal vault was oversewn and left open, to prevent formation of pelvic lymphocysts or hematomas.

The postoperative recovery was uneventful and the patient was discharged from the hospital seven days postoperatively. She received a subcutaneous dose (3500IU) of a low molecular weight heparin (tinzaparin) for her entire hospital stay, starting from the afternoon before surgery was performed. A single 1 gram intravenous dose of cefazolin was administered half an hour before the start of the operation. During her postoperative stay, no antibiotic coverage was provided. The pathology report showed no evidence of lymphovascular space involvement, no extension to the parametrial tissues, clear vaginal margins and all lymph nodes (nine on the left and eleven on the right pelvic side) were negative for tumor metastasis. Further treatment with chemotherapy or radiation therapy was not recommended.

Five weeks after surgery, she presented to the emergency department complaining of right lower quadrant abdominal pain with occasional fevers for two weeks. The patient was unable to move or flex her right hip and had a swollen right groin. The pain was also radiating to the right leg and required oral morphine.

Her vital signs included a temperature of $39.4{ }^{\circ} \mathrm{C}$, blood pressure of $125 / 82 \mathrm{mmHg}$, heart rate of 102 beats per minute and respiratory rate of 13 . On physical examination, the patient was alert, awake and oriented to self, time and place. The cardiovascular exam revealed mild tachycardia with regular rhythm. Lungs were clear to auscultation bilaterally. The abdominal examination showed a well-healed midline infraumbilical incision, no evidence of rigidity or rebound tenderness and presence of normal bowel sounds. A tender palpable mass (measuring $5 \mathrm{~cm}$ in diameter) was noted in the right iliac fossa. No erythema or echymosis were present on the overlying skin. Pelvic and rectal examination did not reveal any abnormalities. Upon examination of the extremities, flexion of the right hip joint with resistance, abduction and extension were extremely painful with decreased range of motion. Deep tendon reflexes were normal bilaterally. Palpation of the femoral, popliteal and dorsalis pedis arteries revealed normal pulses bilaterally. She had no urinary symptoms.

Laboratory investigations included a complete blood count with a White Blood Cell (WBC) count of 14.5 $\mathrm{x} 10^{\wedge} 9 / \mathrm{L}$ with $81 \%$ neutrophils, $\mathrm{Hb}$ of $7.5 \mathrm{~g} / \mathrm{dl}$, Platelets 404 x10^9/L, Sodium $138 \mathrm{mmol} / \mathrm{L}$, Potassium $4.2 \mathrm{mmol} / \mathrm{L}$, Urea $5.5 \mathrm{mmol} / \mathrm{L}$, Creatinine $74 \mathrm{mmol} / \mathrm{L}$, Albumin $29 \mathrm{~g} / \mathrm{L}$, Alanine Transaminase (ALT) $14 \mathrm{u} / \mathrm{L}$, Alkaline Phosphatase (ALP) $185 \mathrm{u} / \mathrm{L}$, Gamma glutamyltransferase (gamma GT) 91 $\mathrm{u} / \mathrm{L}$, Prothrombin time (PT) 21 and Partial thromboplastin time (aPTT) 48. Her C-Reactive Protein (CRP) was 468 $\mathrm{mg} / \mathrm{L}$ (Table 1). Two sets of blood cultures were obtained as well as a urine culture.

Table 1. Blood Results on Admission

\begin{tabular}{|c|c|c|c|}
\hline Blood Results & Value & Normal Range & \\
\hline Sodium & 138 & $134-147$ & $\mathrm{mmol} / \mathrm{L}$ \\
\hline Potassium & 4.2 & $3.5-5.0$ & $\mathrm{mmol} / \mathrm{L}$ \\
\hline Chloride & 106 & $96-106$ & $\mathrm{mmol} / \mathrm{L}$ \\
\hline Urea & 5.5 & $2.5-7.5$ & $\mathrm{mmol} / \mathrm{L}$ \\
\hline Creatinine & 74 & $50-100$ & $\mathrm{Umol} / \mathrm{L}$ \\
\hline Bilirubin & 10 & $<20$ & $\mathrm{Umol} / \mathrm{L}$ \\
\hline Total Protein & 69 & $60-80$ & $\mathrm{~g} / \mathrm{L}$ \\
\hline Albumin & 29 & $34-50$ & $\mathrm{~g} / \mathrm{L}$ \\
\hline ALT & 14 & $5-30$ & $\mathrm{u} / \mathrm{L}$ \\
\hline ALP & 185 & $30-130$ & $\mathrm{u} / \mathrm{L}$ \\
\hline Gamma GT & 91 & $5-40$ & $\mathrm{u} / \mathrm{L}$ \\
\hline CRP & 468 & $<6$ & $\mathrm{mg} / \mathrm{L}$ \\
\hline WBC & 14.5 & $4-11$ & $10 * 9 / \mathrm{L}$ \\
\hline Haemoglobin & 7.5 & $11.5-16.5$ & $\mathrm{~g} / \mathrm{dL}$ \\
\hline Haematocrit & 0.24 & $0.37-0.47$ & \\
\hline Platelets & 404 & $150-400$ & $10 * 9 / \mathrm{L}$ \\
\hline Neutrophils & 11.7 & $2.0-7.5$ & $10 * 9 / \mathrm{L}$ \\
\hline PT & 21 & $10-15$ & $\mathrm{~s}$ \\
\hline aPTT & 48 & $22-35$ & $\mathrm{~s}$ \\
\hline Fibrinogen & 7.4 & $1.5-4.5$ & $\mathrm{~g} / \mathrm{L}$ \\
\hline
\end{tabular}

Imaging studies included a chest $\mathrm{x}$-ray that was unremarkable, without any effusion or infiltrates. Computed tomography (CT) scan of the abdomen and pelvis demonstrated a large $(10 \mathrm{~cm}$ diameter) right psoas muscle mass extending along the entire length of the muscle and a large cavity seen in the lower part of the abdomen, with right hydronephrosis and hydroureter. The presence of a welldefined hypodense lesion with an air-fluid level, in the right psoas muscle is evident (Fig. 1). The appendix was normal with no sign of inflammation, although loops of small bowel were closely applied to the medial surface of the right psoas mass. Differential diagnosis included infected lymphocyst, hematoma and urinoma. The patient was administered intravenous empirical antibiotic treatment with cefuroxime initially and subsequently amoxycillin. A decision was made 


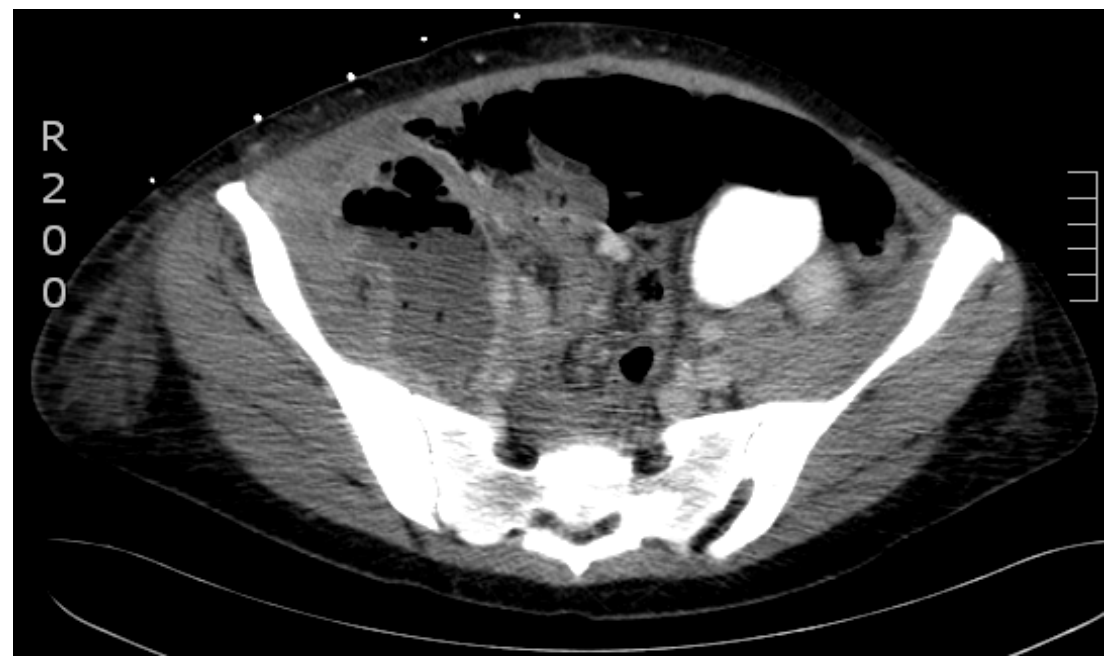

Fig. (1). CT image. Well-defined hypo-dense lesion in the right psoas muscle with peripheral enhancement $(10 \mathrm{~cm})$.

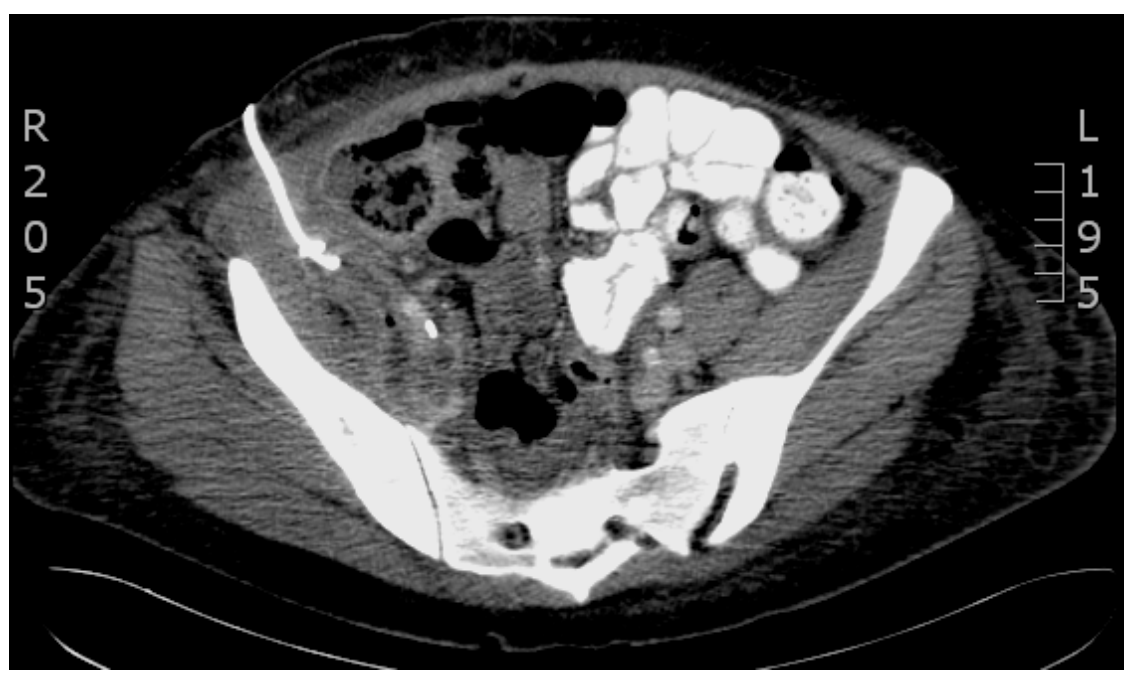

Fig. (2). CT image. Same lesion in the right psoas muscle reduced in size after placement of a drain.

to proceed to percutaneous drainage of the abscess by an interventional radiologist. After the positioning of an intraabdominal drain in the radiology department, $100 \mathrm{ml}$ of thick pus were drained and sent for culture, further $600 \mathrm{ml}$ of pus was drained over the next 48 hours (Fig. 2). The fever and tachycardia resolved within the next $36 \mathrm{hrs}$.

The blood cultures obtained on admission were negative as well as the urine culture. The cultures obtained during drainage of the abscess grew E. Coli and Haemolytic streptococcus group $C$. The antibiotic regimen was adjusted to iv piperacillin/tazobactam and metronidazole according to sensitivity studies. The drain stayed in situ for six days and the patient recovered without further complications. She was discharged home after removal of the catheter on oral coamoxyclav and metronidazole for 4 weeks.

\section{DISCUSSION}

Clinical diagnosis of a psoas abscess is challenging since the symptoms and signs can be variable and nonspecific. The majority of patients present with a non-specific history of weight loss, limp, anorexia, inguinal mass, low back or abdominal pain and fever $[2,11,20-23]$. Leukocytosis $(>10,000 / \mathrm{mL})$ is observed in up to $83 \%$ of cases, elevated $\mathrm{CRP}$ and anemia $<11 \mathrm{~g} / \mathrm{L}(42.6 \%$ in one series) are quite common. Thrombocytosis is less frequently observed $(27 \%$ of cases) [7]. CRP is commonly elevated and on average 189.8 in one series [2]. Aspartate amino-transferase was elevated in $38 \%$ of cases $(>40)$ [7].

The symptomatology and blood test results in our case were very similar to those previously described, although the history and physical examination alone can be identical in patients with pelvic abscesses, peritonitis from other causes such as hematoma, lymphocyst or a urinoma. In the case of an infected lymphocyst, this appears a few days after the operation and may grow steadily. On rectal examination, it would be palpable as a smooth, tense mass attached to the pelvic sidewall. Often the patient is febrile in the immediate postoperative period. Our patient did not present with such a symptomatology. Given the reasonably limited blood loss, the good hemostasis at the end of the operation and the open vaginal vault, the presence of a large hematoma was less probable. Also, in our patient, the pelvis was not reperito- 
nized; significantly reducing the incidence of lymphocyst or hematoma formation.

The main imaging studies, to aid with the diagnosis, are ultrasound and CT scan of the abdomen and pelvis with a sensitivity of $41-95 \%$ and $95-100 \%$ respectively [11]. The most common CT feature of an abscess is a focal hypodense lesion within a larger muscle. Other specific characteristics are the presence of air-fluid level or gas bubbles and irregular margins of the lesion [24]. CT can also identify a retroperitoneal or intra-abdominal disease process as the cause for the abscess. It also allows the simultaneous drainage of the abscess by percutaneous needle aspiration $[11,25,26]$.

Blood cultures and material from the abscess should be sent to the microbiology laboratory for Gram stain and culture. They are positive in 41 to 68 percent of cases; S. Aureus been the most frequent isolate [3, 27]. In our patient, both blood cultures were negative, suggesting a secondary cause, more probably the bowel.

The treatment is based on drainage of the abscess and antibiotics [26]. Secondary abscesses may also require management of the infected focus. The antibiotic treatment should be directed empirically initially against the most common bacteria involved in the process. The appropriate antibiotic treatment should be guided later from blood culture results and culture of the abscess. If there is no significant improvement with intravenous antibiotic treatment, repeating the culture of the abscess and seeking the advise of microbiologist is a valid option. The drainage of the abscess can be done either under image guidance (CT or ultrasound) or through a laparotomy. Percutaneous drainage is much less invasive and useful in cases of cysts that contain few septa [28]. Antibiotic treatment should be continued for 2 to 3 weeks after improvement of the clinical condition and drainage of the abscess. Only a small number of cases reported were treated successfully with antibiotics alone [29].

In our case, the patient's general condition improved dramatically after the drainage of the abscess. Our patient initially received an empiric course of antibiotics based on the suspected clinical diagnosis. The regimen was adjusted to culture results and sensitivities. We decided to proceed with percutaneous drainage of the abscess secondary to the significant size on CT scan and the persistent fevers despite antibiotic treatment. An open approach with drainage of the abscess in the operating room was avoided as an initial approach, since the patient was septic and had recently undergone laparotomy, increasing the risk of postoperative complications [30].

\section{CONCLUSION}

There are few cases of reported psoas abscesses in obstetrics and gynaecology. To our knowledge, this is the first reported case after a gynecological oncology procedure. A psoas abscess should always be suspected after a procedure involving retroperitoneal dissection in a patient who presents with the classical triad of fever, abdominal pain and thigh pain on movement. In the majority of the cases however, the general symptomatology will be nonspecific.
The clinical suspicion should be higher in lengthy oncologic procedures that include more extensive tissue dissections and thus higher infection rates. Even if the source of infection is not initially evident, the clinician must act quickly and aggressively to prevent further complications.

\section{ABBREVIATIONS}

$\begin{array}{ll}\text { ALT } & =\text { Alanine transaminase } \\ \text { ALP } & =\text { Alkaline phosphatase } \\ \text { CT } & =\text { Computed tomography } \\ \text { CRP } & =\text { C-Reactive protein } \\ \text { Gamma GT } & =\text { Gamma glutamyltransferase } \\ \text { PT } & =\text { Prothrombin time } \\ \text { aPTT } & =\text { Partial thromboplastin time } \\ \text { WBC } & =\text { White blood cell }\end{array}$

\section{REFERENCES}

[1] Santaella RO, Fishman EK, Lipsett PA. Primary vs. secondary iliopsoas abscess. Presentation, microbiology and treatment. Arch Surg 1995; 130(12): 1309-13.

[2] Mückley T, Schütz T, Kirschner M, Potulski M, Hofmann G, Bühren V. Psoas abscess: the spine as a primary source of infection. Spine (Phila Pa 1976) 2003; 28(6): E106-13.

[3] Ricci MA, Rose FB, Meyer KK. Pyogenic psoas abscess: worldwide variations in etiology. World J Surg 1986; 10: 834-43.

[4] Buttaro M, González Della Valle A, Piccaluga F. Psoas abscess associated with infected total hip arthroplasty. J Arthroplasty 2002; 17(2): 230-4

[5] Gruenwald I, Abrahamson J, Cohen O. Psoas abscess: case report and review of the literature. J Urol 1992; 147(6): 1624-6.

[6] Mallick IH, Thoufeeq MH, Rajendran T P. Iliopsoas abscesses. Postgrad Med J 2004; 80: 459-62.

[7] López VN, Ramos JM, Meseguer V, et al. Microbiology and outcome of iliopsoas abscess in 124 patients. Medicine (Baltimore) 2009; 88: 120-30.

[8] Lin SS, Vaccaro AR, Reisch S, et al. Low-velocity gunshot wounds to the spine with an associated transperitoneal injury. J Spinal Disord 1995; 8(2): 136-44.

[9] Walsh TR, Reilly JR, Hanley E, et al. Changing etiology of iliopsoas abscess. Am J Surg 1992; 163: 413-6.

[10] Saylam K, Anaf V, Kirkpatrick C. Successful medical management of multifocal psoas abscess following cesarean section: report of a case and review of the literature. Eur J Obstet Gynecol Reprod Biol 2002; 102: 211-4.

[11] Thomas A, Albert AS, Bhat S, et al. Primary psoas abscessdiagnostic and therapeutic considerations. Br J Urol 1996; 78: 35860.

[12] Pasternack MS, Swartz MN. Principles and practice of infectious diseases, $6^{\text {th }}$ ed. New York (NY): Churchill Livingstone 2005; pp. $1200-1$.

[13] Hibbard LT, Snyder EN, McVann RM. Subgluteal and retropsoal infection in obstetric practice. Obstet Gynecol 1972; 39(1): 137-50.

[14] Svancarek W, Chirino O, Schaefer G Jr, Blythe JG. Retropsoas and subgluteal abscesses following paracervical and pudendal anesthesia. JAMA 1977; 237(9): 892-4.

[15] Shah PN, Rane VA, Moolgaoker AS. Retroperitoneal abscess complicating a normal delivery. Br J Obstet Gynaecol 1992; 99(2): 160-1.

[16] Segal S, Gemer O, Sestopal-Epelman M, et al. Retroperitoneal abscess after normal delivery. A report of two cases. J Reprod Med 1996; 41(4): 276-8.

[17] Schutter EM. Psoas abscess in pregnancy: a case report. Geburtshilfe Frauenheilkd 1991; 51(6): 489-90.

[18] Kawamura K, Sekiguchi K, Shibata S, Fukuda J, Tanaka T. Primary psoas abscess during pregnancy. Acta Obstet Gynecol Scand 2000; 79(2): 151-2. 
[19] Scheepers NJ, van Bommel PF, Bleker OP. Psoas abscess related to spontaneous abortion, intra-uterine contraceptive device and curettage. Acta Obstet Gynecol Scand 1993; 72(3): 223-4.

[20] Lipsett PA. Psoas abscess. Schlossberg D, Ed. Current therapy of infectious disease, 2nd ed. St. Louis: Mosby 2001; pp. 255-9.

[21] Bagul NB, Abeysekara AM, Jacob S. Primary psoas abscess due to Streptococcus milleri. Ann Clin Microbiol Antimicrob 2008; 7: 7.

[22] Chern CH, Hu SC, Kao WF, et al. Psoas abscess: making an early diagnosis in the ED. Am J Emerg Med 1997; 15: 83-8.

[23] Naveen K, Senthilkumar A, Nijhawan R, et al. Metastatic carcinoma of cervix mimicking psoas abscess on imaging: a case report. J Gynecol Oncol 2009; 20(2): 129-31.

[24] Zissin R, Gayer G, Kots E, et al. Iliopsoas abscess: a report of 24 patients diagnosed by CT. Abdom Imaging 2001; 26: 533-9.
[25] Torres GM, Cernigiliaro JG, Abbitt PL, et al. Iliopsoas compartment: normal anatomy and pathologic processes. Radiographics 1995; 15: 1285-97.

[26] Gupta S, Suri S, Gulati M, et al. Ilio-psoas abscesses: percutaneous drainage under image guidance. Clin Radiol 1997; 52: 704-7.

[27] Maron R., Levine D, Dobbs TE, Geisler WM. Two cases of pott disease associated with bilateral psoas abscesses: case report. Spine (Phila Pa 1976) 2006; 31: E561.

[28] Dinc H, Onder C, Turhan AU, et al. Percutaneous drainage of tuberculous and nontuberculous psoas abscesses. Eur J Radiol 1996; 23: 130-4.

[29] Gordin F, Stamler C, Mills J. Pyogenic psoas abscesses: noninvasive diagnostic techniques and review of the literature. Rev Infect Dis 1983; 5: 1003-11.

[30] Shuster PA, Barter J.F, Potkul RK, et al. Radical hysterectomy morbidity in relation to age. Obstet Gynecol 1991; 78(1): 77-9.

(C) Spiliopoulos et al.; Licensee Bentham Open.

This is an open access article licensed under the terms of the Creative Commons Attribution Non-Commercial License (http://creativecommons.org/licenses/by$\mathrm{nc} / 3.0 /$ ), which permits unrestricted, non-commercial use, distribution and reproduction in any medium, provided the work is properly cited. 